\title{
TWO NEW SPECIES OF THE GENUS PLATYSEIUS (ACARI: MESOSTIGMATA: BLATTISOCIIDAE) FROM EGYPT
}

\author{
Abd-Elrady Nasr $^{1 *}$, Reham Abo-Shnaf ${ }^{2}$ and Faten Momen ${ }^{1}$ \\ ${ }^{1}$ Pests and Plant Protection Department, National Research Center, Dokii, Giza, Egypt \\ ${ }^{2}$ Plant Protection Research Institute, Agricultural Research Center, Dokii, Giza, Egypt \\ *corresponding author; e-mail: abdelradynasr@yahoo.com
}

\begin{abstract}
The study of Eichhornia spp. in the Sohag and Qualyubia Provinces, Egypt, revealed two new species of the genus Platyseius (family Blattisociidae), Platyseius aegypticus sp. n. and Platyseius girgaensis sp. n. Descriptions are provided based on the morphology of adult females and males. The possible use of these predatory mites as biological control agents should further be investigated.
\end{abstract}

KEY WORDS: Taxonomy, morphology, Gamasina, Platyseius, predators, Eichhornia spp

DOI: 10.21684/0132-8077-2017-25-1-61-73

\section{INTRODUCTION}

Mites of the genus Platyseius Berlese are known to be found in humid habitats (Moraes et al. 2015). They were mentioned as possible predators of mosquitoes, as they were found on adult mosquitoes and flies (Reisen and Mullen 1978; Smith 1983; Halliday et al. 1998). The presence of some elongated and usually strip-like setae on tarsi II-IV, the shape and size of their paradactyli, and the median projection of the pulvilli on these legs can be considered as adaptations to move in moist habitats or water films (Evans and Hyatt 1960; Karg 1971; Lindquist 2003). The thickened peritremes in these mites, which extend posteriad to the hind edge of the peritrematal shields, suggest their function as a plastron (Hinton 1971). The genus Platyseius Berlese, 1916 includes about 20 described species reported from Asia, Africa, Australia, Europe, North America, and South America (Schrank 1803; Berlese 1905, 1916; Halbert 1923; Willmann 1938; Evans and Hyatt 1960; Krantz 1962; Bhattacharyya 1968; Ishikawa 1969; Karg 1971, 1977, 1993, 1994, 2006; Halliday et al. 1998; Lindquist 2003; Kazemi and Rajaei 2013; Kazemi et al. 2016; Moraes et al. 2016). Lindquist (2003) divided the genus Platyseius into two species groups: the subglaber species group is distinguished by the presence of only two of the five pairs of $J$-setae ( $J 1-J 3$ missing); setae $Z 5$ thicker than adjacent setae; metapodal platelets fused to peritrematal shields; ventrianal shield with 5-6 pairs of opisthogastric setae in addition to circumanal setae, and the italicus species group that is distinguished by the presence of three pairs of $J$ setae $(J 1, J 2$ absent); setae $Z 5$ slender; metapodal platelets free; ventrianal shield with 3-4 pairs of opisthogastric setae in addition to circumanal setae (rarely with two or four pairs).

The objective of the present work is to describe two new species from the two species groups of the genus Platyseius.

\section{MATERIAL AND METHODS}

Different mite samples were collected from the Qualyubia and Sohag provinces on the water plant Eichhornia spp. (Pontederiaceae). Mites were mounted in Hoyer's medium for later examination. Descriptions were done with phase contrast (Olympus, BHA) and (Nikon, Eclipse E400) microscopes.

Illustrations were made with the use of an eyepiece attached to the phase contrast microscope and measured with the use of a graded ocular for description. Notation of body setae is based on Lindquist and Evans (1965), with the modifications proposed by Lindquist (1994). Measurements are given in micrometers $(\mu \mathrm{m})$ for each structure. Coordinates provided are approximate, as they were not taken at the time when the samples were collected.

\section{THE SUBGLABER SPECIES GROUP}

Platyseius aegypticus Nasr et Abo-Shnaf, sp.n.

Figs. 1-20

Diagnosis. Dorsal shield mostly smooth, with a scantly short-lined striae around the muscle scars, unsclerotised lateral cuticle with five pairs of $r-R$ setae; presternal region with two pairs of transversely elongate platelet; sternal shield with scantly sparse anterolateral striae, genital shield smooth, ventrianal shield elliptical, with a transverse line anteriad of anal opening; opisthogaster with two 


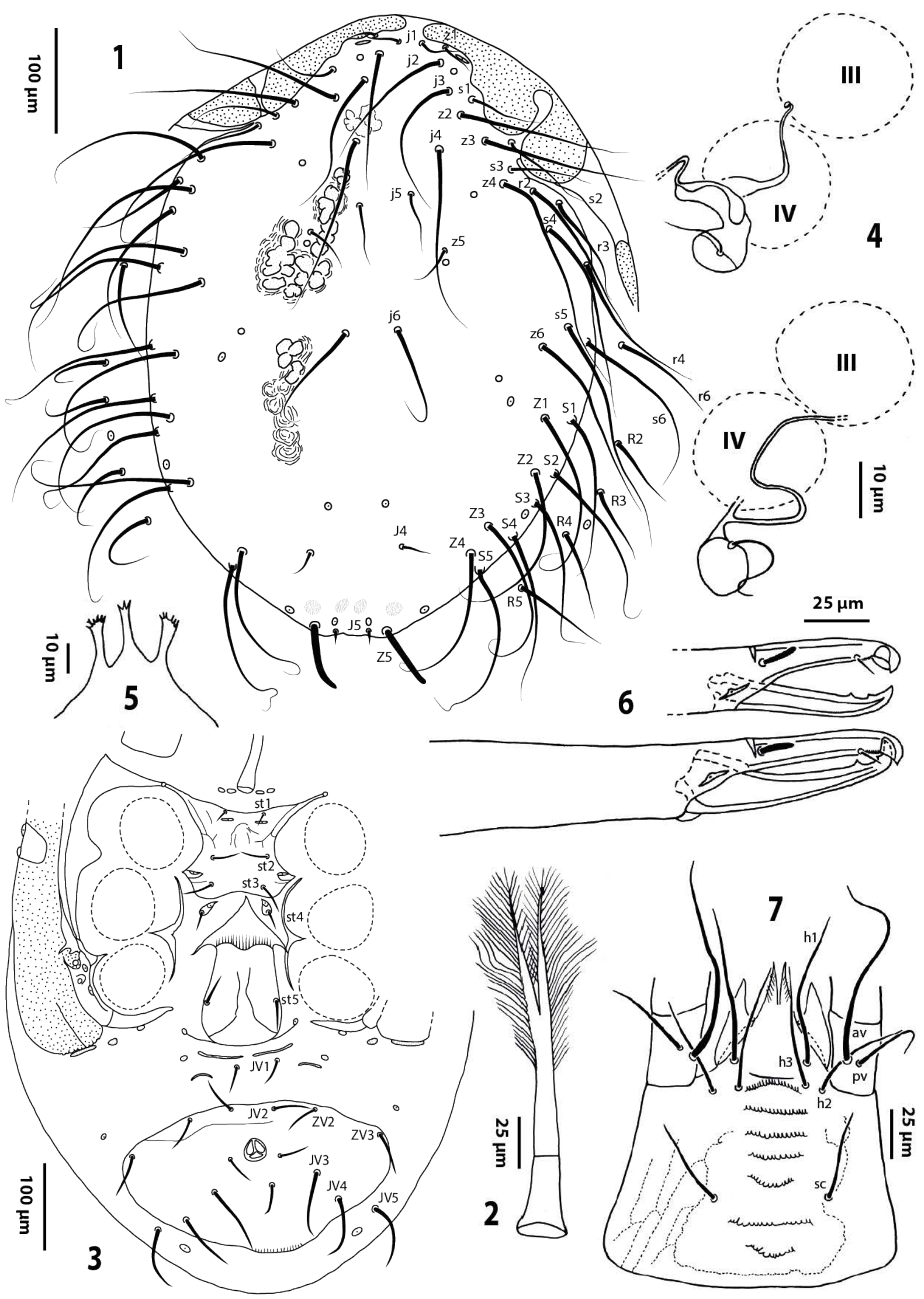

Figs. 1-7. Platyseius aegypticus sp. n. female (holotype): 1-dorsum of idiosoma, 2-tritosternum, 3-venter of idiosoma, 4-spermathecae, 5-epistome, 6-chelicerae, 7-hypostome. 

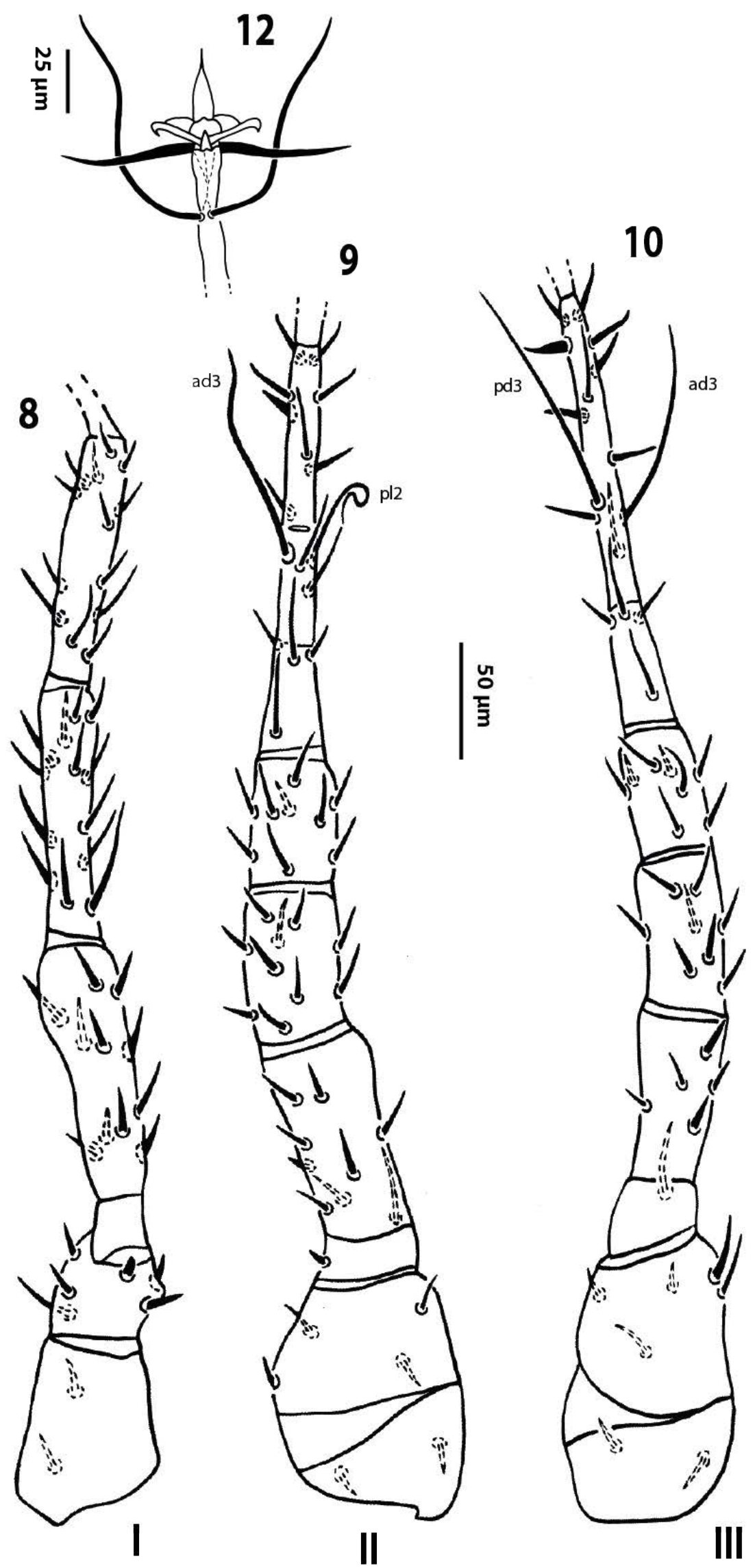

11
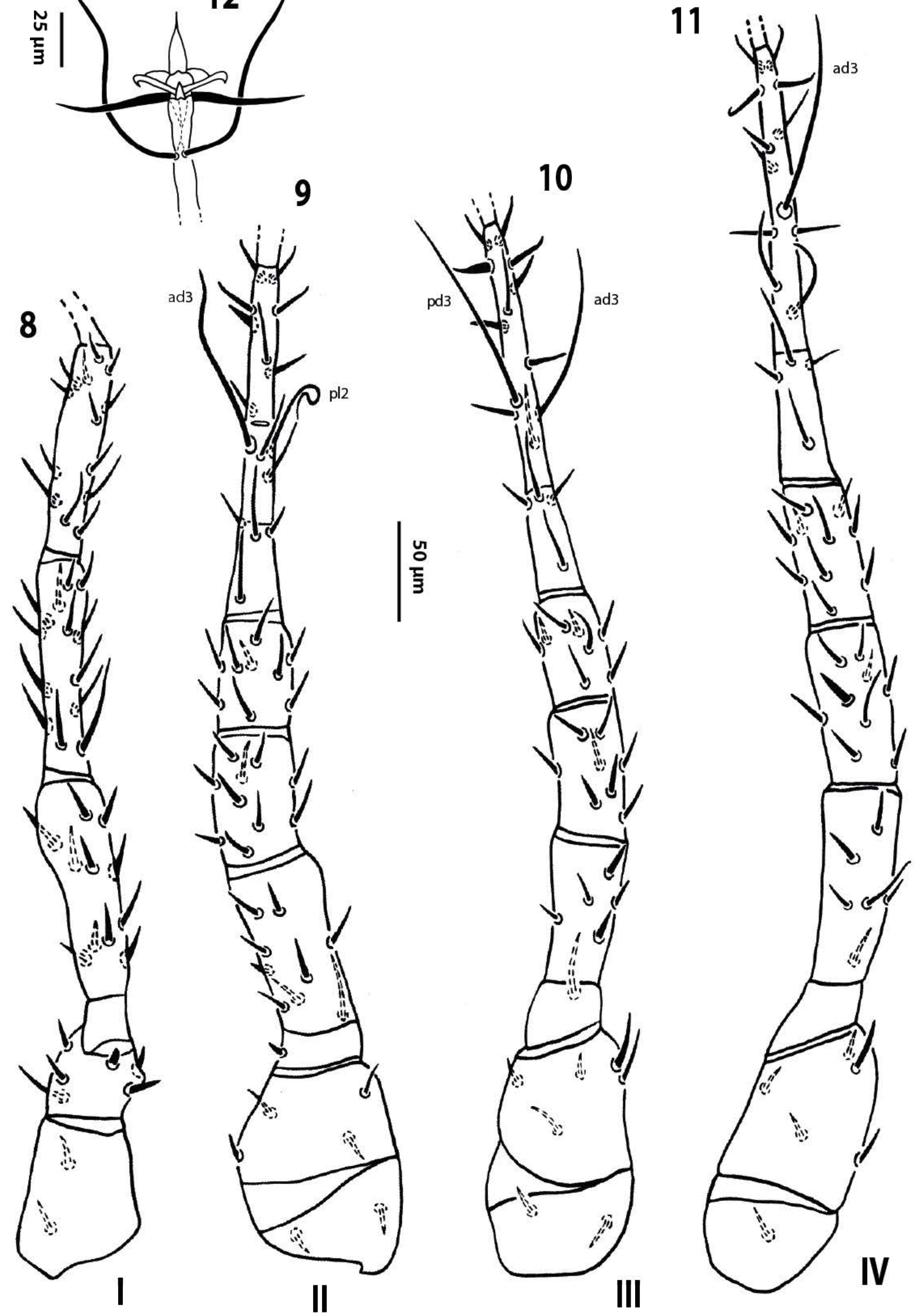

Figs. 8-12. Platyseius aegypticus sp. $n$. female (holotype): 8 - coxa-tibia of leg I, 9-coxa-tarsus of leg II, 10-coxatarsus of leg III, 11—coxa-tarsus of leg IV, 12—pretarsus of leg IV. 


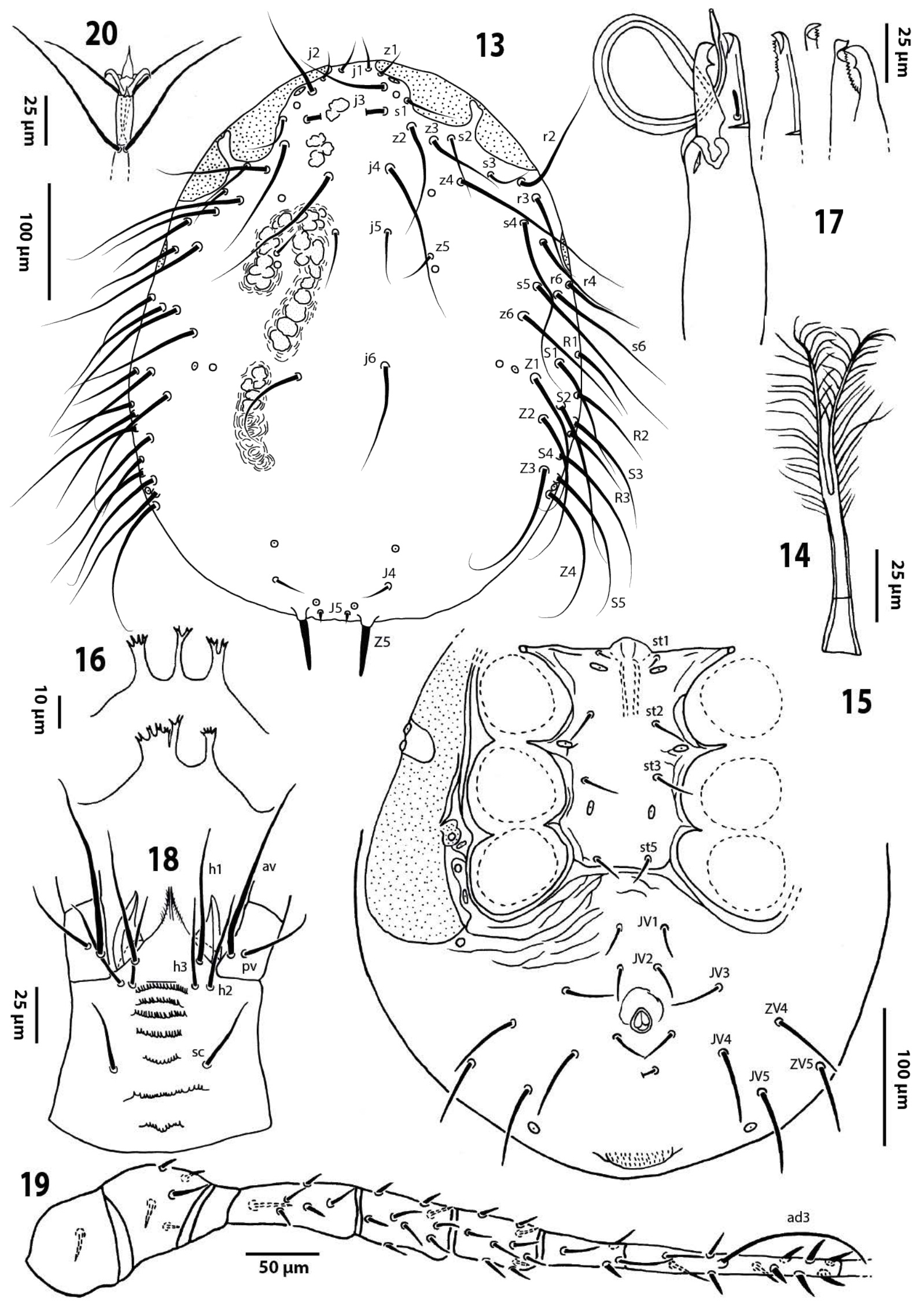

Figs. 13-20. Platyseius aegypticus sp. n. male (paratype): 13-dorsum of idiosoma, 14-tritosternum, 15-venter of idiosoma, 16-epistomes, 17-chelicerae, 18-hypostome, 19-coxa-tarsus of leg IV, 20-pretarsus of leg IV. 
pairs of setae on unsclerotized cuticle; with two pairs of metapodal plates; spermathecal apparatus distinguishable as a lightly sclerotized tubular section may be the major duct, leading from the insemination pore, apparently opening next to anterior margin of coxa IV, distinguishable; section connected to the major duct, which may be the atrium and calyx, gives rise to a short, fine minor duct; anteromedian region of epistome three-tined, the median one with three denticles, the two laterals with 6-7 denticles each; fixed cheliceral with about six minute teeth; movable digit with two teeth; legs II-IV with elongate and strip-like setae on telotarsus.

Description. Adult female (two specimens measured; Figs. 1-12).

Dorsum of idiosoma (Fig. 1). Dorsal shield 644, 635 long and 446, 441 wide at widest level. Podonotal region of the dorsal shield mostly smooth, with a scantly short-lined striae around the muscle scars, with 21 pairs of setae $(j 1-j 6, z 1-z 6$, $s 1-s 6, r 2-r 4)$, a pair of distinguishable lyrifissures and three pairs of distinguishable pores. Opisthonotal region mostly smooth, with a scantly shortlined striae around the muscle scars, with 12 pairs of setae $(J 4, J 5, Z 1-Z 5, S 1-S 5)$, five pairs of distinguishable lyrifissures and a pair of distinguishable pores. Most dorsal shield setae of uniform length and shape and longer than distance to respective subsequent setae $(j 5, j 6, z 5$ and $J 4$ shorter than distance to respective subsequent setae), flagellate and smooth, except Z5 stout and blunt-tiped ( $j 1$, $z 1, J 4$ and $J 5$ shortest). Unsclerotised lateral cuticle with five pairs of setae $(r 6, R 2-R 5)$, all aciculate, and with a lyrifissure $(R p)$ between $R 3$ and $R 4$. Setal lengths: $j 1$ 49, 47; $j 2135,130 ; j 3143,138$; j4 205, 195; j5 60; j6 125; z1 42; z2 188, 185; z3 166, 161; z4 242, 239; z5 47, 42; z6 213, 208; s1 70; s2 88, 83; s3 83, 81; s4 239, 234; s5 241, 237; s6 202, 192; r2 199, 190; r3 112, 104; r4 153, 151; r6 99, 91; J4 39, 36; J5 10; Z1 229, 226; Z2 208; Z3 211, 205; Z4 192, 187; Z5 66, 65; S1 187, 183; S2 202, 190; S3 156, 153; S4 185, 182; S5 205, 200; $R 2$ 78, 65; R3 81; R4 86, 60; R5 91, 75.

Venter of idiosoma (Figs. 2, 3). All setae aciculate and smooth. Base of tritosternum 41 long and 21 wide at medium level; laciniae 167 long, divided for about $48,46 \%$ of their total length. Presternal region with two pairs of transversely elliptical platelets. Sternal shield fused with anterior portion of endopodal plate, with a pair of pores (gstl) on distal end of extension between coxae I-II, with scantly sparse anterolateral striae; 127 , 122 long and 120, 112 wide at level of st2, with three pairs of setae (st1-st3) and two pairs of lyrifissures; posterior margin concave. Posterior portion of endopodal plate represented by a large triradiate fragment between coxae III-IV. Metasternal plates ellipse-shaped with the fourth pair of sternal seta (st4) and a pair of lyrifissures (iv3). Genital shield smooth, 199, 198 long, including the hyaline flap; with posteriorly divergent lateral margins; posterior margin convex, 100, 99 wide at posterior corners, bearing genital setae (st5); distance between genital setae 86, 85; paragenital lyrifissures (iv5) on unsclerotized cuticle, posterolaterad of st5. Ventrianal shield elliptical, with a transverse line anteriad of anal opening, 194, 185 long and 338, 324 wide at widest level, with five pairs of opisthogastric setae (JV2-JV4 and ZV2, ZV3) in addition to circumanal setae; anal opening small, 34 , 31 long including frame. With $J V 1$, a continues line, a pair of transversely platelets; a pair of transversely concave platelets and a pair of lyrifissures between genital and ventrianal shields. With two pairs of punctate metapodal plates, the anterior plate smaller, oval mediad of the posterior one, elongate, apparently fused with peritrematic plate. Unsclerotized cuticle laterad and posteriad of ventrianal shield with a pair of setae (JV5) and two pairs of distinguishable lyrifissures. Exopodal plate distinct from posterior margin of coxa IV to anterior margin of coxa II; with a pore near posterior end $[S J$, inguinal solenostome of Athias-Henriot (1969), gv2 of Lindquist and Moraza (2008)]. Setal lengths: st1 12; st2 29; st3 33; st4 21; st5 31; JV1 29; JV2 47; JV3 78, 75; JV4 94, 72; JV5 75, 70; ZV2 43, 42; ZV3 49; para-anal 33, 31 and postanal 39,36 .

Peritrematic plate and peritreme (Figs. 1, 3). Peritrematic plate fused with dorsal shield at level of $s 3$; broadly fused with exopodal plate between coxae III-IV and around coxa IV; with a lyrifissure and a pore in region between coxae II-III (ip2, $g p 1)$, with two lyrifissures and a pore behind stigma ( $i p 3$, $i p 4, g p 2$ ). Peritreme extending forward to level of $j 1$.

Spermatheca (Fig. 4). Spermathecal apparatus distinguishable as a lightly sclerotized tubular section may be the major duct about 203 long, leading from the insemination pore, apparently opening at posterior margin of coxa III, distinguishable; terminating in sclerotized globular pouch, gives rise to a short, fine minor duct.

Gnathosoma (Figs. 5-7). Anteromedian region of epistome three-tined, the median one with three denticles, the two laterals with 6-7 denticles each. 
Cheliceral antiaxial and dorsal lyrifissures and dorsal seta as well distinct. Fixed digit 112, 109 long, with a apical notch, apparently fitting inner margin of movable digit, and with about six minute teeth and setiform pilus dentilis; movable digit 107 long, with two teeth, the anterior minute. Deutosternum with eight free-standing transverse lines, the first (most distal) of which smooth; from second to eighth lines with respectively $35,35,33,33,20$, 25 and 18 denticles each, the seventh row slightly wider than others. Corniculi parallel to each other, about 55, 52 long and 20, 18 wide at the base. Setal lengths: $h 1$ 91, 88; $h 2$ 60, 57; $h 3$ 70, 68 and sc 53, 52; palp trochanter setae $a v 112,109$ and $p v 49,47$.

Legs (Figs. 8-12). Pretarsi I-IV each with a pair of claws and median lobe of pulvillus slender. Leg lengths: I-711, 707; II—621; III—644, 635 and IV-779, 774. Leg chaetotaxy-femora I lacking $p v 1$ and $p v 2$, femora II lacking $a v 1$; genu: $\mathrm{I}-2$, $3 / 2,2 / 1,2$; II $-2,3 / 1,2 / 0,2$; III-2, 2/1, 2/0, 1; IV $-2,2 / 1,3 / 0$, 1; tibia: I-2, 3/2, 3/1, 2; II -2 , 2/1, 2/0, 2 ( $p v$ missing); III-2, 1/1, 2/1, 1; IV-2, $1 / 1,3 / 1,2$. Leg II with two spine-like setae on telotarsus ( $a d 3, p l 2)$ 117, 114; 91, 90; leg III with two spine-like setae on telotarsus $(a d 3, p d 3) 86$, 83; 120, 113; leg IV with a spine-like setae on telotarsus (ad3) 146, 143.

Adult male (two specimens measured; Figs. 13-20).

Dorsum of idiosoma (Fig. 13). Dorsal shield 450, 448 long and 365 wide. Podonotal and opisthonotal regions of the dorsal shield mostly smooth, with a scantly short-lined striae around the muscle scars. Podonotal region with 22 pairs of setae ( $j 1-$ $j 6, z 1-z 6, s 1-s 6, r 2-r 4, r 6)$, a pair of distinguishable lyrifissures and three pairs of distinguishable pores. Opisthonotal region with 15 pairs of setae $(J 4, J 5, Z 1-Z 5, S 1-S 5, R 1-R 3)$, four pairs of distinguishable lyrifissures and a pair of distinguishable pores. Relative lengths and shapes of setae as in adult female. Unsclerotized lateral cuticle with no setae. Setal lengths: $j 1$ 34, 31; j2 91, 88; j3 (broken); j4 172; j5 49, 47; j6 117, 112; z1 31, 29; z2 140; z3 120, 114; z4 182, 179; z5 36, 34; z6 153, 151; s1 49, 47; s2 62, 60; s3 47, 44; s4 179, 177; s5 182, 177; s6 151; r2 140, 137; r3 86, 83; r4 107, 96; r6 81, 79; J4 31; J5 8; Z1 166, 164; Z2 166, 164; Z3 159, 148; Z4 164, 159; Z5 49, 48; S1 143, 138; S2 172, 164; S3 138; S4 166, 164; S5 161;R1 73, 69; $R 2$ 87, 78; $R 3$ 73, 68.

Venter of idiosoma (Figs. 14, 15). Shape of setae as in adult female. Base of tritosternum 23, 21 long and 13 wide at medium level; laciniae 120 long, divided for about $55 \%$ of their total length. Sternogenital shield fused with whole endopodal plate, with a pair of pores (gstl) on distal end of extension between coxae I-II, with anterolateral striae, smooth elsewhere; 192, 191 long and 100, 99 wide at level of $s t 2$, with four pairs of setae (st1-st3, st5; st4 suppressed) and three pairs of lyrifissures. Ventrianal shield fused with whole exopodal plate, reticulate anteriad of anal opening, 222, 217 long and 378, 374 wide at anterior corners, with seven pairs of opisthogastric setae (JV1-JV5 and $Z V 4, Z V 5$ ) in addition to the circumanal setae and with a pair of distinguishable lyrifissures; anal opening small, 26 long including frame. Exopodal plate distinct from posterior margin of coxa IV to anterior margin of coxa II. Setal lengths: st 1 10, 9; st2 31; st3 33, st 5 23; JV1 26; JV2 23; JV3 36; JV4 62; JV5 73, 65; ZV4 68, 52; ZV5 60; para-anal 31 and post-anal (broken).

Peritrematic plate and peritreme (Figs. 13, 15). Peritrematic plate fused with whole dorsal shield; broadly fused with exopodal plate between coxae III-IV; with a lyrifissure and a pore in region between coxae II-III (ip2, gp 1), with two lyrifissures and a pore behind stigma ( $i p 3, i p 4, g p 2)$. Peritreme extending forward to level between $j 1$ and $z 1$.

Gnathosoma (Figs. 16-18). Anteromedian region of epistome three-tined, the median one with 3-4 denticles, the two laterals with five denticles (in one specimen, left side with nine denticles). Cheliceral antiaxial and dorsal lyrifissures and dorsal seta as well distinct. Fixed digit 55 long, with three teeth in apical notch, apparently fitting inner margin of movable digit between those teeth, and with 11-12 teeth and setiform pilus dentilis; movable digit 60, 57 long, with a single tooth and twisted spermadactyl, about 221, 216 long. Deutosternum with eight free-standing transverse lines, the first (most distal) of which smooth; from second to eighth lines with respectively $30,25,30,32,18$, 28 and 14 denticles each, the seventh row slightly wider than others. Corniculi parallel to each other, about 42, 40 long and 14, 13 wide at the base. Setal lengths: $h 1$ 73, 70; $h 2$ 55, 52; $h 3$ 52, 49; sc 42; palp trochanter setae $a v 91,88$ and $p v$ 44, 42.

Legs (Figs. 19, 20). Legs similar to those of adult female. Leg lengths: I-599, 594; II-522, 513; III-531, 522 and IV-653, 648. Leg chaetotaxy as in adult female. Leg II with two spine-like setae on telotarsus ( $a d 3, p l 2)$ 117, 112; 75, 73; leg III with two spine-like setae on telotarsus ( $a d 3$, $p d 3)$ 94, 91; 114, 91; leg IV with a spine-like setae on telotarsus (ad3) 122, 117. 
Type specimens. Holotype female from Eichhornia spp., January 27, 2008, at Sandanhour (31 ${ }^{\circ}$ $11^{\prime}$ E, $\left.30^{\circ} 24^{\prime} \mathrm{N}\right)$, Banha, Qualyubia Province; one paratype male from the same substrate, March 30, 2008, at Berket el-Sabe'e ( $\left.31^{\circ} 5^{\prime} \mathrm{E}, 30^{\circ} 38^{\prime} \mathrm{N}\right)$, Monufia Province, deposited in the mite reference collection of Acarological Unit, Pests and Plant Protection Department, National Research Center, Dokii. One paratype female from the same substrate, January 27, 2008, at Sandanhour, Banha, Qualyubia Province; one paratype male from the same substrate, March 30, 2008, at Berket el-Sabe'e, Monufia Province, deposited in the mite reference collection of Zoology and Agricultural Nematology Department, the Faculty of Agriculture, Cairo University, all collected by Kh. M. Saleh.

Etymology. The name aegypticus is a composition "aegypt" derived from Egypt (the country where the type specimens were collected) + icus, a suffix denoting "belonging to".

Remarks. This new species is most similar to Platyseius triangralis Ishikawa, 1969 and Platyseius persicus Kazemi, Payandeh and Saberi, 2016. The former differs from the new species by having dorsal shield much shorter, 508 long according to the original description; setae $j 1, j 4$ and $z 1$ are much shorter about respectively $(0.6,0.5,0.7)$ times as long as the corresponding measurements of our new species, while setae $j 3, s 2, s 4, j 6$ ( $J 1$ as reported) are much longer about respectively $(2.1,2.3,1.2$, 1.1) times as long as the corresponding measurements of our new species; posterior portion of endopodal plate represented by two tri-radiate fragments between coxae II-III and III-IV; ventrianal shield much smaller, 162 long and 290 wide according to the original description; seta $Z V 3$ much shorter; a pair of transversely platelets between genital and ventrianal shields much shorter; with a pair of metapodal plates; median region of epistome with three denticles; fixed cheliceral digit edentate. The latter differs by having dorsal shield with 32 pairs of setae ( $z 6$ missing); unsclerotised lateral cuticle with four pairs of setae $(r 5, R 1, R 4, R 5)$; most dorsal shield setae much shorter; $J 4$ stout; fixed cheliceral digit with ca. 12-15 minute teeth.

\section{THE ITALICUS SPECIES GROUP}

\section{Platyseius girgaensis Nasr et Momen, sp. $\mathbf{n}$.}

Figs. 21-40

Diagnosis. Dorsal shield reticulate, unsclerotised lateral cuticle with three pairs of $r-R$ setae; presternal region smooth; sternal shield with scantly sparse lateral striae, genital shield smooth, ventrianal shield with transverse reticulations; opisthogaster with six pairs of setae on unsclerotized cuticle; with a pair of punctate metapodal plates; spermathecal apparatus distinguishable as a lightly sclerotized tubular section may be the major duct leading from the insemination pore, apparently opening next to anterior margin of coxa IV, distinguishable; section next to insemination pore, which may be the atrium and calyx, gives rise to a long, fine minor duct; anteromedian region of epistome three-tined, the median one longer and with two denticles, the two laterals with three denticles each; fixed cheliceral digit edentate; movable digit with two teeth; legs II-IV with elongate and strip-like setae on telotarsus.

Description. Adult female (two specimens measured; Figs. 21-32).

Dorsum of idiosoma (Fig. 21). Dorsal shield 420, 412 long and 299, 265 wide at widest level. Podonotal region of the dorsal shield reticulate, with 21 pairs of setae $(j 1-j 6, z 1-z 6, s 1-s 6, r 2-r 4)$, four pairs of distinguishable lyrifissures and three pairs of distinguishable pores. Opisthonotal region reticulate, with 14 pairs of setae $(J 2-J 5, Z 1-Z 5$, $S 1-S 5$ ) (in the holotype, left $J 2$ missing), four pairs of distinguishable lyrifissures and three pairs of distinguishable pores. Most dorsal shield setae of uniform length and shape and shorter than distance to respective subsequent setae (setae $j 2, J 3$ longer than distance to respective subsequent setae), aciculate and smooth ( $j 1$ and $J 5$ shortest). Unsclerotized lateral cuticle with three pairs of setae (r6, R1 and $R 3)$, all aciculate, and with a lyrifissure $(R p)$ posteriad of $R 3$. Setal lengths: $j 19,8 ; j 221$, 18 ; 3329,23 ; $j 421$; j5 22, 21; j6 34, 31; z1 21, 16; z2 29, 23; z3 29; z4 30, 29; z5 16; z6 31, 29; s1 29, 26; s2 34, 31; s3 34, 31; s4 34, 29; s5 31; s6 34, 29; r2 29; r3 34, 31; r4 34, 31; r6 31, 29; J2 38, 34; J3 31, 30; J4 34, 29; J5 13, 12; Z1 36, 31; Z2 30, 29; Z3 29; Z4 29; Z5 30, 29; S1 33, 31; S2 31, 29; S3 31, 29; S4 30, 29; S5 29; R1 31, 26; R3 30, 29.

Venter of idiosoma (Figs. 22, 23). All setae aciculate and smooth. Base of tritosternum 38, 35 long and 14 wide at medium level; laciniae 124, 117 long, divided for about $45,42 \%$ of their total length. Presternal region smooth. Sternal shield fused with anterior portion of endopodal plate, with a pair of pores ( $g s t 1)$ on distal end of extension between coxae I-II, with scantly sparse lateral striae; 96, 87 long and 82 wide at level of st2, with three pairs of setae (st1-st3) and two pairs of lyrifissures; posterior margin concave. Posterior portion 


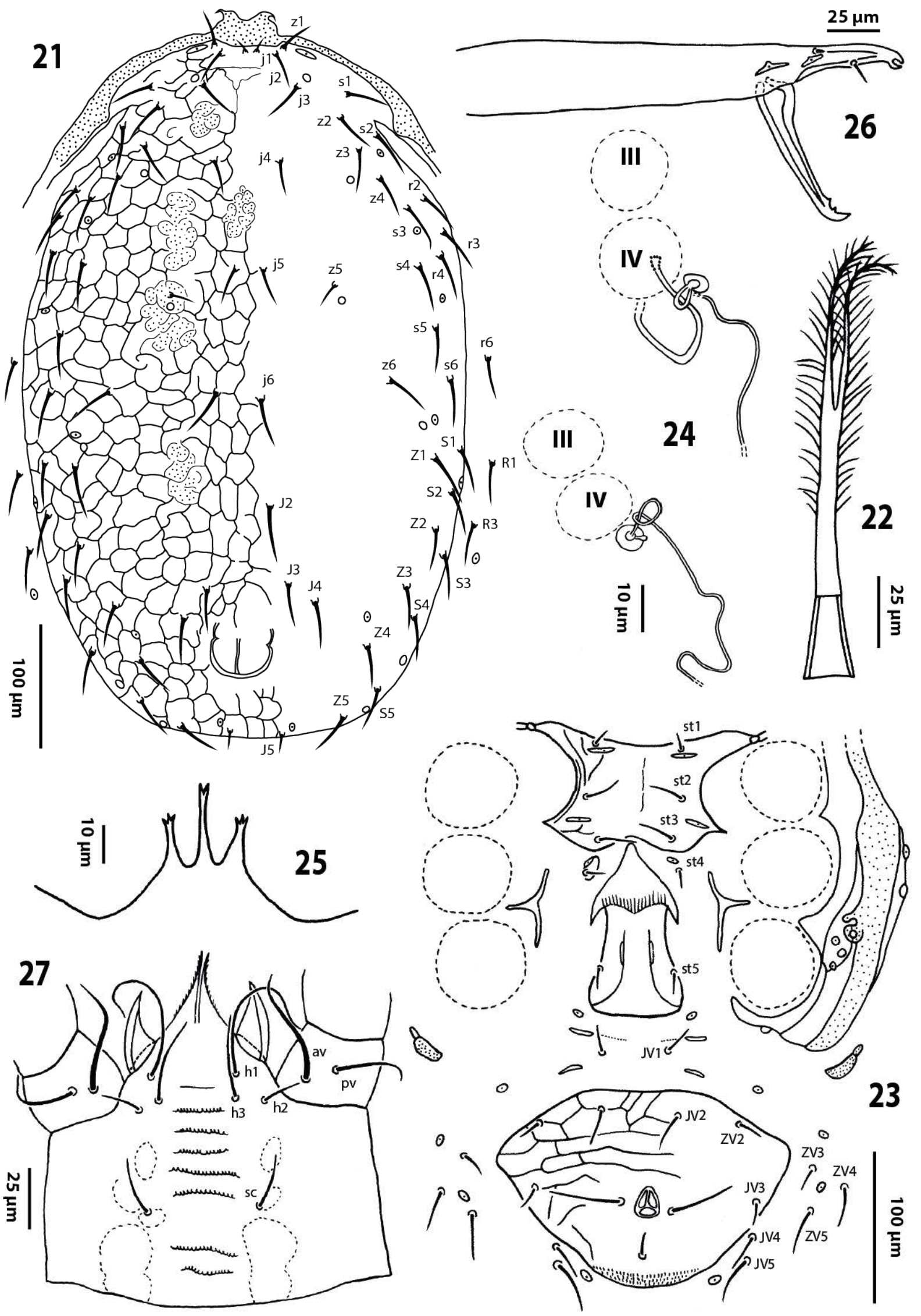

Figs. 21-27. Platyseius girgaensis sp. n. female (holotype): 21-dorsum of idiosoma, 22-tritosternum, 23-venter of idiosoma, 24-spermathecae, 25-epistome, 26-chelicera, 27-hypostome. 


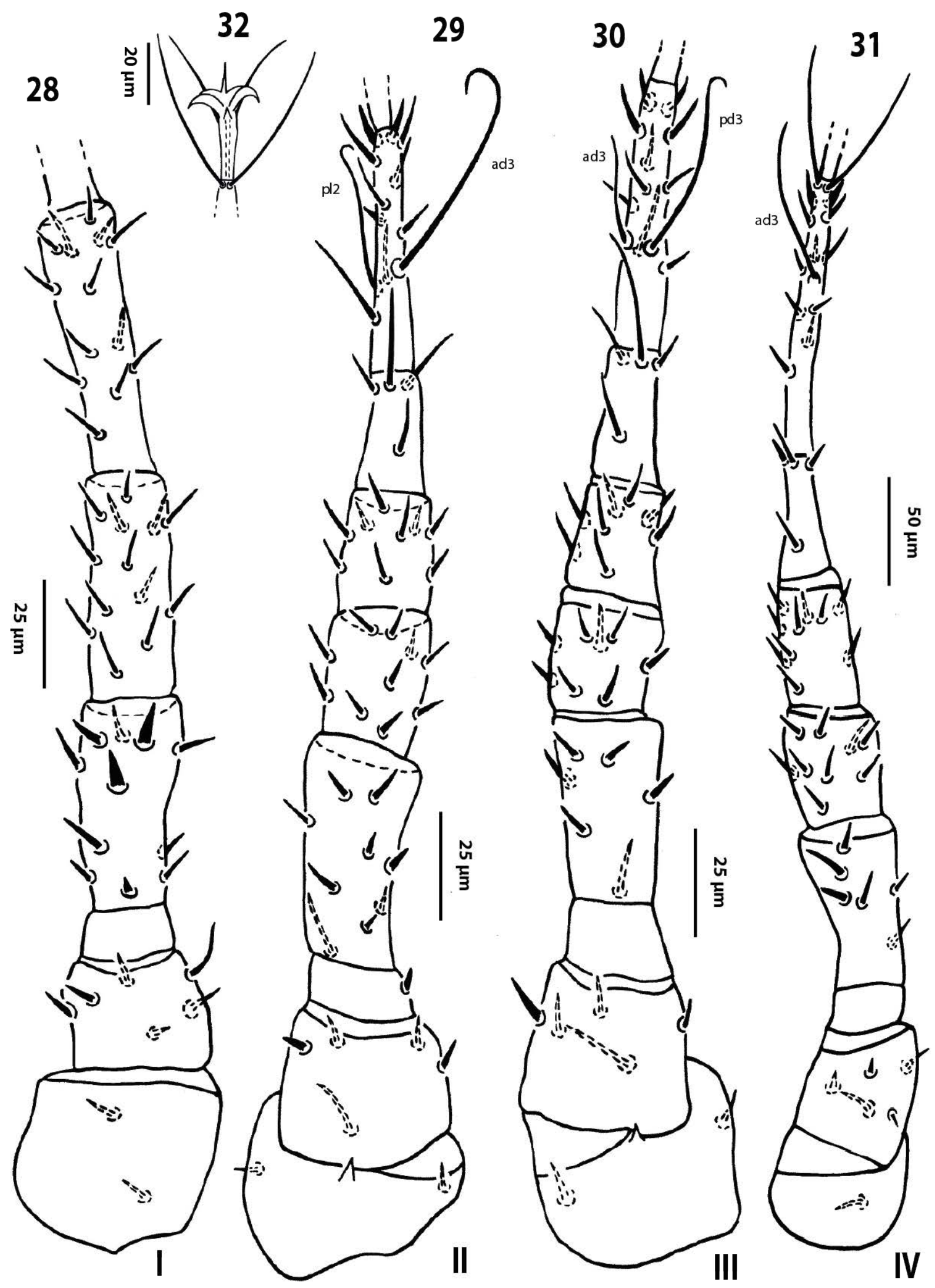

Figs. 28-32. Platyseius girgaensis sp. n. female (holotype): 28 - coxa-tibia of leg I, 29-coxa-tarsus of leg II, 30coxa-tarsus of leg III, 31-coxa-tarsus of leg IV, 32-pretarsus of leg IV. 


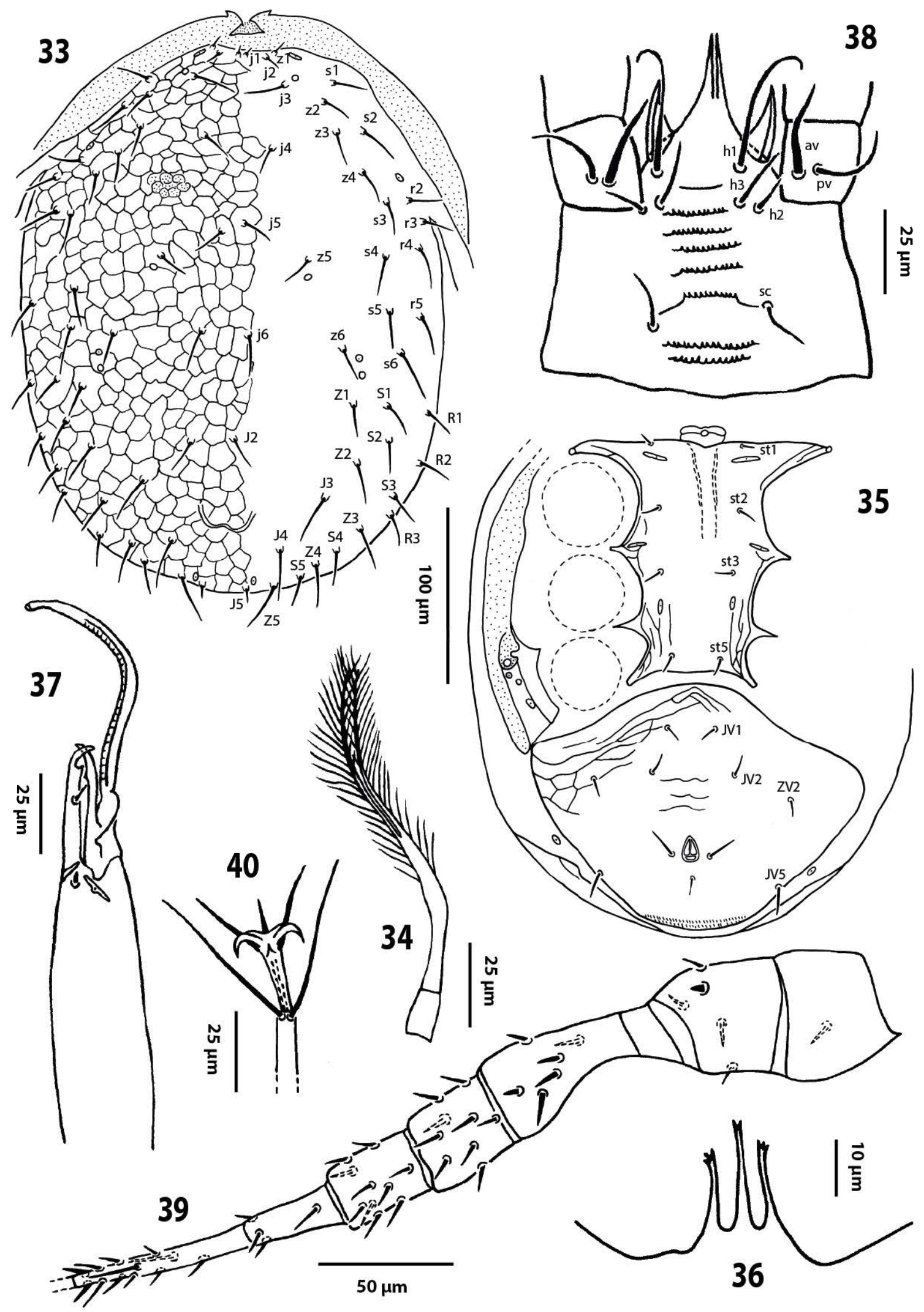

Figs. 33-40. Platyseius girgaensis sp. n. male (paratype): 33-dorsum of idiosoma, 34-tritosternum, 35-venter of idiosoma, 36-epistome, 37-chelicera, 38-hypostome, 39-coxa-tarsus of leg IV, 40-pretarsus of leg IV. 
of endopodal plate represented by a large tri-radiate fragment between coxae III-IV. Metasternal plates elliptical-shaped with the fourth pair of sternal seta (st4) and a pair of lyrifissures (iv3) (in the holotype, right metasternal plate missing). Genital shield smooth, 130, 127 long, including the hyaline flap; with posteriorly divergent lateral margins; posterior margin slightly truncate, 70, 65 wide at posterior corners, bearing genital setae (st5); distance between genital setae 53, 52; paragenital lyrifissures (iv5) on unsclerotized cuticle, posterolaterad of st5. Ventrianal shield subtriangular, with transverse reticulations, 127, 112 long and 189, 176 wide at widest level, with three pairs of opisthogastric setae $(J V 2, J V 3$ and $Z V 2)$ in addition to circumanal setae; anal opening small, 20, 18 long including frame. With $J V 1$, a broken line with barely distinguishable interruptions, two pairs of transversely elongate platelets and a pair of lyrifissures between genital and ventrianal shields. With a pair of punctate metapodal plates. Unsclerotized cuticle laterad and posteriad of ventrianal shield with five pairs of setae (JV4, JV5 and ZV3-ZV5) and three pairs of distinguishable lyrifissures. Exopodal plate distinct from posterior margin of coxa IV to anterior margin of coxa II; with a pore near posterior end [SJ, inguinal solenostome of Athias-Henriot (1969), gv2 of Lindquist and Moraza (2008)]. Setal lengths: st 1 8; st2 23, 21; st3 23, 22; st4 13, 11; st5 18, 17; JV1 21, 20; JV2 22, 21; JV3 18, 16; JV4 22, 17; JV5 27, 26; ZV2 18, 16; ZV3 18, 16; ZV4 23; ZV5 26, 25; para-anal 33, 30 and post-anal 14 .

Peritrematic plate and peritreme (Figs. 21, 23). Peritrematic plate fused with dorsal shield at level anteriad of $s 2$, anterior extremity of peritremes continuous and indented below vertex; broadly fused with exopodal plate between coxae III-IV and around coxa IV; with a lyrifissure and a pore in region between coxae II-III ( $i p 2, g p 1)$, with two lyrifissures and a pore behind stigma (ip3, ip4, gp2).

Spermatheca (Fig. 24). Spermathecal apparatus distinguishable as a lightly sclerotized tubular section may be the major duct leading from the insemination pore, apparently opening next to anterior margin of coxa IV, distinguishable; section next to insemination pore, which may be the atrium and calyx about ( 5 in diameter), gives rise to a long, fine minor duct.

Gnathosoma (Figs. 25-27). Anteromedian region of epistome three-tined, the median one longer and with two denticles, the two laterals with three denticles each. Cheliceral antiaxial and dorsal lyrifissures and dorsal seta as well distinct. Fixed digit 62 long, edentate, with apical notch, apparently fitting inner margin of movable digit; and setiform pilus dentilis; movable digit 65 long, with two teeth. Deutosternum with eight free-standing transverse lines, the first (most distal) of which smooth; from second to eighth lines with respectively 18, 22, 20, 30, 18, 25 and 20 denticles each, the fifth to seventh rows slightly wider than others. Corniculi parallel to each other, about 36, 35 long and 14,13 wide at the base. Setal lengths: $h 175$, 73 ; $h 217,16$; $h 335,33$ and $s c 30,26$; palp trochanter setae $a v$ 57, 55 and $p v$ 35, 34.

Legs (Figs. 28-32). Pretarsi I-IV each with a pair of claws and median lobe of pulvillus slender. Leg lengths: I-467, 460; II-394, 359; III-385, 354 and IV - 493, 484. Leg chaetotaxy-genu: I- 2, 3/2, 3/1, 2; II - 2, 3/1, 2/0, 2; III-2, 2/1, 2/0, 1; IV-2, 2/1, 3/0, 1; tibia: I-2, 3/2, 3/1, 2; II-2, 1/1, 2/1, 2 (ad2 missing); III-2, 1/1, 2/1, 1; IV-2, 1/1, 3/1, 2. Leg II with two spine-like setae on telotarsus $(a d 3, p l 2) 70,68 ; 47,44$; leg III with two spine-like setae on telotarsus $(a d 3, p d 3) 44$, $42 ; 73,72$; leg IV with a spine-like setae on telotarsus (ad3) 73, 68 .

Adult male (one specimen measured) (Figs. 33-40).

Dorsum of idiosoma (Fig. 33). Dorsal shield 320 long and 239 wide. Podonotal and opisthonotal regions of the dorsal shield reticulate. Podonotal region with 22 pairs of setae $(j 1-j 6, z 1-z 6$, $s 1-s 6, r 2-r 5)$, two pairs of distinguishable lyrifissures and two pairs of distinguishable pores. Opisthonotal region with 17 pairs of setae $(J 2-J 5$, $Z 1-Z 5, S 1-S 5, R 1-R 3)$. Relative lengths and shapes of setae as in adult female. Unsclerotized lateral cuticle with no setae. Setal lengths: $j 15, j 213, j 3$ 26, j4 16, j5 18, j6 26, z1 10, z2 18, z3 23, z4 21, $z 518, z 620, s 121, s 226, s 322, s 426, s 525, s 6$ 31, r2 22, r3 21,r4 26, r5 26, J2 23, J3 31, J4 31, J5 10, Z1 23, Z2 26, Z3 27, Z4 27, Z5 29, S1 23, $S 2$ 23, S3 23, S4 23, S5 22, R1 21, R2 21, R3 21.

Venter of idiosoma (Figs. 34, 35). Shape of setae as in adult female. Base of tritosternum 14 long and 9 wide at medium level; laciniae 118 long, divided for about $49 \%$ of their total length. Sternogenital shield fused with whole endopodal plate, with a pair of pores (gst 1) on distal end of extension between coxae I-II, with lateral and posterior striae, smooth elsewhere; 126 long and 65 wide at level of $s t 2$, with four pairs of setae $(s t 1-s t 3$, st5) (st4 suppressed) and three pairs of lyrifissures. Ventrianal shield subtriangular, reticulate anteriad of anal opening, 138 long and 185 wide at anterior corners, 
with four pairs of opisthogastric setae $(J V 1, J V 2$, $J V 5$ and $Z V 2$ ) in addition to the circumanal setae; anal opening small, 18 long including frame. Unsclerotized cuticle laterad and posteriad of ventrianal shield with a pair of distinguishable lyrifissures. Exopodal plate distinct from posterior margin of coxa IV to anterior margin of coxa II. Setal lengths: st1 8, st2 13, st3 13, st5 13, JV1 13, JV2 14, JV5 18, ZV2 13, para-anal 22 and post-anal 13.

Peritrematic plate and peritreme (Figs. 33, 35). Peritrematic plate fused with dorsal shield at level of $r 3$, anterior extremity of peritremes continuous and indented below vertex; broadly fused with exopodal plate between coxae III-IV; with two lyrifissures and a pore behind stigma (ip3, $i p 4, g p 2)$.

Gnathosoma (Figs. 36-38). Anteromedian region of epistome three-tined, the median one longer and with two denticles, the two laterals with three denticles each. Cheliceral antiaxial and dorsal lyrifissures and dorsal seta as well distinct. Fixed digit 40 long, edentate, with apical notch, apparently fitting inner margin of movable digit; and setiform pilus dentilis; movable digit 42 long, apparently with a single tooth and curved spermadactyl, about 79 long. Deutosternum with eight freestanding transverse lines, the first (most distal) of which smooth; from second to eighth lines with respectively $18,20,20,20,15,25$ and 18 denticles each, the sixth row slightly wider than others. Corniculi parallel to each other, about 25 long and 9 wide at the base. Setal lengths: $h 149, h 216, h 3$ 25, sc 21; palp trochanter setae $a v 31$ and $p v 29$.

Legs (Figs. 39, 40). Legs similar to those of adult female. Leg lengths: I-380, II-315, III307 and IV-387. Leg chaetotaxy as in adult female. Setae on telotarsus of leg II not possible to measure because of the position; leg III with two spine-like setae on telotarsus ( $a d 3, p d 3) 34,55$; leg IV with a spine-like setae on telotarsus ( $a d 3) 59$.

Type specimens. Holotype female and one paratype male from Eichhornia spp., September 1, 1979, at Girga ( $31^{\circ} 53^{\prime}$ E, $\left.26^{\circ} 20^{\prime} \mathrm{N}\right)$, Sohag Province, deposited in the mite reference collection of Acarological Unit, Pests and Plant Protection Department, National Research Center, Dokii. One paratype female from the same substrate, locality and date, deposited in the mite reference collection of Zoology and Agricultural Nematology Department, the Faculty of Agriculture, Cairo University, all collected by A.K. Nasr.

Etymology. The specific name girgaensis refers to "from Girga", the Egyptian city where the type specimens of this species were collected.
Remarks. The new species is most similar to Platyseius berlesei (Krantz, 1962) and Platyseius cooki Lindquist, 2003. The former differs from the new species by having most of the dorsal shield setae much shorter, epistome with three smooth anterior extensions, fixed and movable digits edentate. The latter differs by having dorsal shield with 33 pairs of setae ( $j 1$ suppressed to alveolar vestiges, $J 2$ missing); fixed cheliceral digit with large subapical tooth followed by ca. 8-12 teeth.

\section{REFERENCES}

Athias-Henriot, C. 1969. Les organes cuticulaires sensoriels et glandulaires des Gamasides. Poroïdotaxie et adénotaxie. Bulletin de la Société Zoologique de France, 94: 485-492.

Berlese, A. 1905. Acari nuovi. Materiali pel "Manipulus V". Redia, 2: 231-238.

Berlese, A. 1916. Centuria prima di Acari nuovi. Redia, 12: 19-67.

Bhattacharyya, S.K. 1968. Studies on Indian mites (Acarina : Mesostigmata). 6. Six records and descriptions of nine new species. Acarologia, 10, 527-549.

Evans, G.O. and Hyatt, K.H. 1960. A revision of the Platyseiinae (Mesostigmata: Aceosejidae) based on material in the collections of the British Museum (Natural History). Bulletin of the British Museum (Natural History), Zoology, 6: 27-101. http://dx.doi.org/10.5962/bhl.part.26844

Halbert, J.N. 1923. Notes on Acari, with descriptions of new species. Journal of the Linnean Society (Zoology), 35: 363-392. http://dx.doi.org/10.1111/ j.1096-3642.1923.tb01732.x

Halliday, R.B., Walter, D.E. and Lindquist, E.E. 1998. Revision of the Australian Ascidae (Acarina : Mesostigmata). Invertebrate Taxonomy, 12: 1-54.

Hinton, H.E. 1971. Plastron respiration in the mite, Platyseius italicus. Journal of Insect Physiology, 17: 1185-1199. http://dx.doi.org/10.1016/00221910(71)90184-3

Ishikawa, K. 1969. Studies on the mesostigmatid mites in Japan. IV. Family Blattisociidae Garman. Reports of Research Matsuyama Shinonome Junior College, 4: 111-139.

Karg, W. 1971. Acari (Acarina), Milben, Unterordnung Anactinochaeta (Parasitiformes): Die freilebenden Gamasina (Gamasides), Raubmilben. In: Die Tierwelt Deutschlands und der angrenzenden Meeresteile, 59. Teil. Gustav Fischer Verlag, Jena, 475 pp.

Karg, W. 1977. Neuearten den raubmilbenfamilie Ascidae Oudemans, 1905 (Acarina, Parasitiformes) aus Chile. Mitteilungen aus dem Zoologischen 
Museum in Berlin, 53: 285-302. http://dx.doi. org/10.1002/mmnz.19770530206

Karg, W. 1993. Acari (Acarina), Milben Parasitiformes (Anactinochaeta) Cohors Gamasina Leach, Raubmilben. In: Die Tierwelt Deutschlands und der angrenzenden Meeresteile nach ihren Merkmalen und nach ihrer Lebensweise. 59. Teil. 2 überarbeitete Auflage, Gustav Fischer Verlag, Jena, Stuttgart, New York, 523 pp.

Karg, W. 1994. Raubmilben der Ascidae, Ameroseiidae, Rhodacaridae und Macrochelidae auf dem Galapagos-Archipel (Acarina, Parasitiformes). Mitteilungen aus dem Zoologischen Museum in Berlin, 70: 113-131. http://dx.doi.org/10.1002/mmnz. 19940700107

Karg, W. 2006. The systematics of Parasitiformes, especially of Gamasina Leach (Acarina), with new species from Ecuador. Mitteilungen aus dem Museum für Naturkunde in Berlin, Zoologische Reihe, 82: 140-169. http://dx.doi.org/10.1002/mmnz. 200600002

Kazemi, S. and Rajaei, A. 2013. An annotated checklist of Iranian Mesostigmata (Acari), excluding the family Phytoseiidae. Persian Journal of Acarology, 2: 63-158.

Kazemi, S., Payandeh, M. and Saberi, S. 2016. A new species of Platyseius Berlese (Acari: Mesostigmata: Blattisociidae) from Iran, and a key to the world species of the genus. Zootaxa, 4139: 566574. http://doi.org/10.11646/zootaxa.4139.4.8

Krantz, G.W. 1962. Acari: free-living Mesostigmata. II.-The family Aceosejidae. Parc National de La Garamba, 34: 3-29.

Lindquist, E.E. 1994. Some observations on the chaetotaxy of the caudal body region of gamasine mites (Acari : Mesostigmata), with a modified notation for some ventrolateral body setae. Acarologia, 35 : 323-326.

Lindquist, E.E. 2003. Observations on mites of the Subfamily Platyseiinae, with description of two new species of Platyseius from North America (Acari: Mesostigmata: Ascidae). In: Smith, I.M. (Ed.). An
Acarological Tribute to David R. Cook (from Yankee Springs to Wheeny Creek). Indira Publishing House, West Bloomfield, MI, pp. 155-182.

Lindquist, E.E. and Evans, G.O. 1965. Taxonomic concepts in the Ascidae, with a modified setal nomenclature for the idiosoma of the Gamasina (Acarina: Mesostigmata). Memoirs of the Entomological Society of Canada, 47, $64 \mathrm{pp}$.

Lindquist, E.E. and Moraza, M.L. 2008. A new genus of flower-dwelling melicharid mites (Acari: Mesostigmata: Ascoidea) phoretic on bats and insects in Costa Rica and Brazil. Zootaxa, 1685: 1-37.

Moraes, G.J. de, Britto, E.P.J., Mineiro, J.L. de C. and Halliday, B. 2016. Catalogue of the mite families Ascidae Voigts \& Oudemans, Blattisociidae Garman and Melicharidae Hirschmann (Acari: Mesostigmata). Zootaxa, 4112: 1-299.

Moraes, G.J. de, Venancio, R., Santos, V.L.V. dos and Paschoal, A.D. 2015. Potential of Ascidae, Blattisociidae and Melicharidae (Acari: Mesostigmata) as biological control agents of pest organisms. In: Carrillo, D. et al., (Eds.). Prospects for Biological Control of Plant Feeding Mites and Other Harmful Organisms. Progress in Biological Control. Springer, 19, p. 33-75. http://dx.doi.org/10.1007/978-3319-15042-0 2

Reisen, W.K. and Mullen, G.R. 1978. Ecological observations on acarine associates (Acari) of Pakistan mosquitoes (Diptera: Culicidae). Entomologia Experimentalis et Applicata, 7: 769-776.

Schrank, F. von P. 1803. Fauna Boica, Durchgedachte Geschichte der in Baiern einheimischen und zahmen Thiere. Philipp Krull, Universitatsbuchhandler, Landshut, $272 \mathrm{pp}$.

Smith, B.P. 1983. The potential of mites as biological control agents of mosquitoes. In: Hoy, M.A., Cunningham, G.L., and Knutson, L. (Eds.). Proceedings of biological control of pests by mites. University of California, Berkeley, 1982, pp. 79-85.

Willmann, C. 1938. Beitrag zur Kenntnis der Acarofauna des Komitates Bars. Annales Musei Nationalis Hungarici, 31: 144-172. 